\title{
Spinocerebellar ataxia type 6 family with phenotypic overlap with Multiple System Atrophy
}

\author{
Rana Hanna Al-Shaikh ${ }^{* 1}$, Anna I. Wernick ${ }^{{ }^{2,4}}$, Audrey J. Strongosky ${ }^{1}$, Alexandra I. Soto-Beasley ${ }^{2}$, \\ Jay A. van Gerpen ${ }^{1,5}$, William P. Cheshire ${ }^{1}$, Ryan J. Uitti ${ }^{1}$, Owen A. Ross ${ }^{2,3}$, Zbigniew K. Wszolek ${ }^{1}$ \\ ${ }^{1}$ Department of Neurology, Mayo Clinic, Jacksonville, Florida, United States \\ ${ }^{2}$ Department of Neuroscience, Mayo Clinic, Jacksonville, Florida, United States \\ ${ }^{3}$ Department of Clinical Genomics, Mayo Clinic, Jacksonville, Florida, United States \\ ${ }^{4}$ University College London, London, United Kingdom \\ ${ }^{5}$ University of Alabama at Birmingham, Huntsville, Alabama, United States
}

\begin{abstract}
Aim of the study. Multiple system atrophy (MSA) and spinocerebellar ataxia (SCA) share similar symptomatology. We describe a rare occurrence of familial MSA that proved to be SCA6 upon genetic analysis.

Materials and methods. Eighty MSA patients were enrolled in our study; blood samples were collected and genetic screening of the familial case for known SCA loci was performed.

Results. A 68-year-old woman presented with recurrent and severe episodes of light-headedness, imbalance, frequent falls, neck and lower back stiffness, subjective arm and leg weakness, and numbness and tingling in both feet. One year later, her condition had declined; she experienced more falls, worsening instability, again more generalised but still subjective weakness, impaired fine motor movements, slurred speech, difficulty swallowing, episodes of choking, bladder incontinence, and constipation. Clinical suspicion included parkinsonism, MSA, and SCA. The patient was enrolled in our MSA study and was found to have 22 and 12 CAG repeats in CACNA1A. The other 79 clinical MSA patients were negative for SCA6 screening.

Conclusions and clinical implications. While MSA and SCA may have similar presentations during early disease stages, the presence of both conditions on the list of differential diagnoses can be a diagnostic dilemma. Further analysis will aid in developing a biomarker to distinguish between the two conditions and guide proper management.
\end{abstract}

Key words: cerebellum, gait disorders/ataxia, SCA, spinocerebellar ataxia

(Neurol Neurochir Pol 2020; 54 (4): 350-355)

\section{Introduction}

Multiple system atrophy (MSA) and spinocerebellar ataxia (SCA) share a similar phenotype, largely due to the involvement of cerebellar degeneration present in both conditions, particularly the cerebellar form of MSA [1]. This means that differentiating between the two conditions can be challenging. SCA is an autosomal dominant neurodegenerative condition that affects the cerebellum and spinal cord. More than 45 genetic subtypes of SCA have been identified and listed in the Online Mendelian Inheritance in Man database at the National Centre for Biotechnology Information. Various types of mutations are known to result in SCA [2-4], including polyglutamine CAG repeat expansion. SCA type 6 (SCA6) is caused by a CAG trinucleotide repeat in the CACNA1A gene. Possessing 20 to 33 CAG repeats in 1 allele on CACNA1A has proven to

"Contributed equally to the manuscript.

Presented at the $144^{\text {th }}$ Annual Meeting of the American Neurological Association, St. Louis, Missouri, October 2019.

Address for correspondence: Zbigniew K. Wszolek, MD, Department of Neurology, Mayo Clinic, 4500 San Pablo Rd, Jacksonville, FL 32224, USA,

e-mail:Wszolek.Zbigniew@mayo.edu 
be pathogenic [5-10]. Our genetic analysis demonstrated the presence of SCA6 in one familial patient among our cohort of 80 patients with MSA.

\section{Materials and methods}

Standard protocol approval, patient consent, clinical scales/assessments, sample collection

The study was approved by the Mayo Clinic Institutional Review Board, and written informed consent was obtained from all participants. Neurological evaluations were performed by movement disorder specialists at the Mayo Clinic Florida, patients were followed clinically by four of the authors (J.A.V.G., W.P.C., R.J.U., and Z.K.W.), and all office visits and outside records were reviewed. Blood samples were collected from all patients. Eighty patients clinically diagnosed with MSA were recruited and enrolled, beginning in 1999 and extending to the present day. The following data was collected: date of birth, sex, race, age at enrollment, age at disease onset, disease duration, family history of neurological conditions, and past medical history. One patient reported a family history of parkinsonism reminiscent of MSA (Fig. 1, subject III-2), and as a result we screened for several genes to verify whether a genetic condition was indeed present. After obtaining the results, we decided to screen the remainder of the cohort $(n=79)$. Diagnostic criteria were adopted from the first MSA consensus in 1998, consisting of four main domains: I Autonomic and Urinary Dysfunction, II Parkinsonism, III Cerebellar Dysfunction, and IV Corticospinal Dysfunction, and were updated after the second consensus in 2008 [11, 12].

\section{Magnetic resonance imaging}

Neuroradiological studies were performed for the index case (subject III-2). This included magnetic resonance imaging (MRI) scans. All MRI sequences obtained for clinical purposes were reviewed (see Supplemental Fig. 1).

\section{Genetic analysis}

Genomic DNA was extracted from whole blood using a FlexSTAR automated extraction machine (AutoGen, Inc) according to the manufacturer's instructions. Whole exome sequencing was performed on our familial MSA patient using an Agilent SureSelect Human All Exon V5+UTRs capture kit, with sequencing performed on an Illumina HiSeq2500 by the Mayo Clinic Genomics Core. Exome data was processed using the Mayo Genome GPS v4.0 pipeline. Functional annotations of variants were performed using ANNOVAR (version 2016Feb01). Genotype calls with $\mathrm{GQ}<10$ and/or depth (DP) $<10$ were set to missing, and variants with $\mathrm{ED}>4$ were removed from all subsequent analyses. For all analyses, only variants that passed Variant Quality Score Recalibration (VQSR) and with a call rate $>95 \%$ were considered, unless otherwise specified. We extracted the identified variants for the SCA-associated genes from the generated VCF using Golden Helix (SNP \& Variation Suite v8.8.3); see Supplemental Table 1. In addition, we screened nine SCA genes with known pathogenic repeats/insertions (again see Supplemental Table 1). The regions of interest were amplified (FAM-labelled forward primer) using standard polymerase chain reaction (PCR) protocol, and the PCR products were run out on an agarose gel. The products were diluted based on the intensity of the gel image. GeneScan 400HD ROX dye Size Standard (Applied Biosystems) was then added to the diluted product, which was read on an ABI 3730xl DNA Sequencer (Applied Biosystems). Allelic sizes were analysed using GeneMapper Software 5 (Applied Biosystems). For Sanger sequence validation of pathogenic repeat length, primers were designed for the CACNA1A repeat, and the region was amplified using standard PCR protocol and cloned using a TOPO $^{\text {mo }}$ TA Cloning ${ }^{\text {mo }}$ Kit (Invitrogen). Products were cleaned using Agencourt AMPure XP magnetic beads and the Biomek FXP Dual Arm System (Beckman Coulter, Inc). The same primers were used for sequencing using a BigDye Terminator Cycle Sequencing Kit (Applied Biosystems). The cycle sequence products were cleaned with Agencourt CleanSEQ magnetic beads (Beckman Coulter, Inc) and read on an ABI 3730xl DNA Sequencer. Sequences were then analysed using SeqScape v3.0 (Applied Biosystems) (Fig. 1).

\section{Results}

Our proband carried a pathogenic $C A G$ trinucleotide repeat in CACNA1A, providing a genetic diagnosis of SCA6 rather than MSA. Genetic analysis revealed $22 \mathrm{CAG}$ repeats on 1 allele and 12 CAG repeats on a second allele of CACNA1A; the longer repeat length was confirmed by Sanger sequencing (Fig. 1). Further screening of our clinical MSA series for SCA6 $(\mathrm{n}=79)$ did not identify any other carriers of a pathogenic CACNA1A repeat length.

\section{Clinical case description}

The patient with SCA6 was a 68 -year-old woman with a history of aortic regurgitation, arterial hypertension, and lacunar infarcts who presented with recurrent and severe episodes of light-headedness, gait imbalance, and frequent falls. She did not seem to think that her falls were due to the chronic dizziness, and cardiovascular work up demonstrated aortic regurgitation. She also complained of stiffness in her neck and lower back, weakness beginning in the arms then involving the legs, and numbness and tingling in both feet. She used a walking stick. She noted that her local neurologist had diagnosed her with Parkinson's Disease and administered the regular formulation of carbidopa/levodopa. However she was unable to tolerate it due to numbness, tingling, and experienced no benefit from it. 


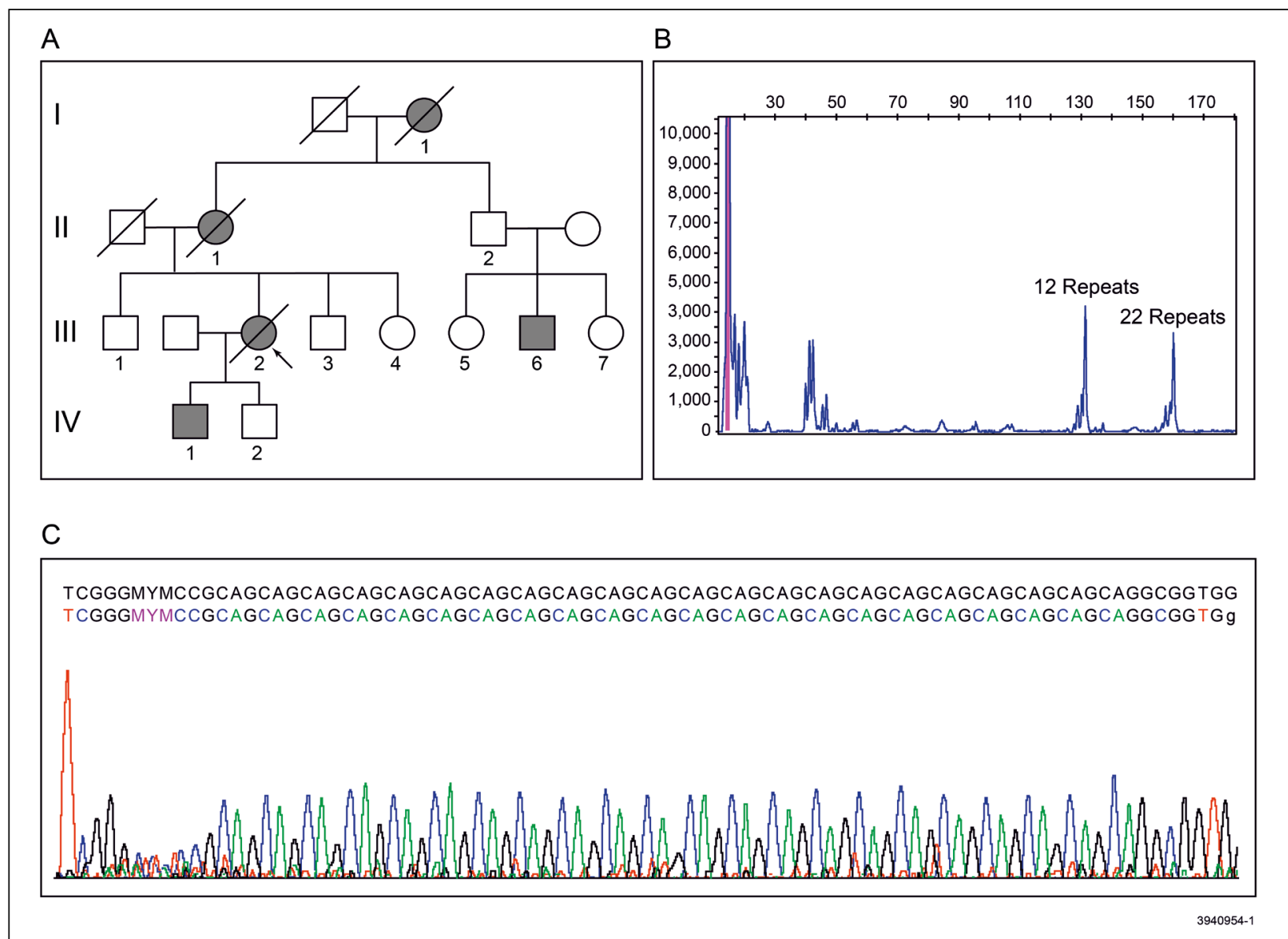

Figure 1. A. Family pedigree of patient with CACNA1A 22 CAG repeats on allele 1, and 12 CAG repeats on allele 2. Pathogenic range is 20 to 33 $\mathrm{CAG}$ repeats. Squares indicate men, circles indicate women; shaded symbols indicate affected individuals. Arrow indicates proband, and slashed line through symbol indicates deceased individual. Individual IV-1 developed gait impairment and underwent neurological evaluation by his local physician at an outside institution. B. Proband (III-2), peaks identified by fluorescent polymerase chain reaction assay, visualised on GeneMapper software, highlighting an expanded repeat. C. Proband (III-2), cloning and sequencing confirmed a 22-repeat allele in the CACNA1A gene

Her maternal grandmother, mother, and a maternal cousin all experienced gait and speech impairment (Fig. 1).

Neurological evaluation demonstrated nystagmus, slow saccades, reduced upgaze, and square wave jerks. Reduced facial expression and drooling were absent. She required assistance in walking, and her Romberg and pull tests were positive. Stooped posture and tremor were absent. Spasticity and hyperreflexia were present in the lower extremities. Muscle strength was normal in the upper and lower extremities. Two to three beats of non-sustained ankle clonus were present, plantar responses were positive bilaterally. Formal assessment for orthostatic hypotension and autonomic reflex screening were not performed.

Nerve conduction studies of right motor and sensory median and ulnar nerves, right tibial, peroneal and sural nerves, and needle examination of selected right upper and lower extremity muscles were normal. Amantadine was prescribed, $100 \mathrm{mg}$ twice a day, and the patient noted a significant improvement in her gait and balance. At her second visit 12 months later, the patient's condition had worsened; she had experienced more falls and she demonstrated worsening of balance, generalised but subjective muscle weakness, impaired fine motor movements, slurred speech, difficulty swallowing, and episodes of choking. She had also developed urinary stress incontinence treated with a pubovaginal sling, as well as constipation. Clinical suspicion included parkinsonism based on her generalised body bradykinesia. Probable MSA was considered based on the presence of urinary dysfunction, parkinsonism with poor levodopa response, and cerebellar dysfunction as per the initial MSA consensus [11]. According to the second consensus, she fulfilled the criteria for possible MSA due to the presence of parkinsonism, cerebellar syndrome, autonomic dysfunction, Babinski sign with hyperreflexia, gait ataxia and postural instability [12]. SCA was added to the list of differentials due to the presence of gait impairment, fine motor dysfunction, in addition to hypophonic and dysarthric speech. A more aggressive trial of carbidopa/levodopa was administered; one half tablet of 25/100 escalated in half-tablet increments each week until reaching 2.5 tablets per dose, three times per day. Total daily dose of levadopa was $750 \mathrm{mg}$. The patient did not report to the clinic after this point. 
Table 1. Clinical characteristics of SCA6 and MSA

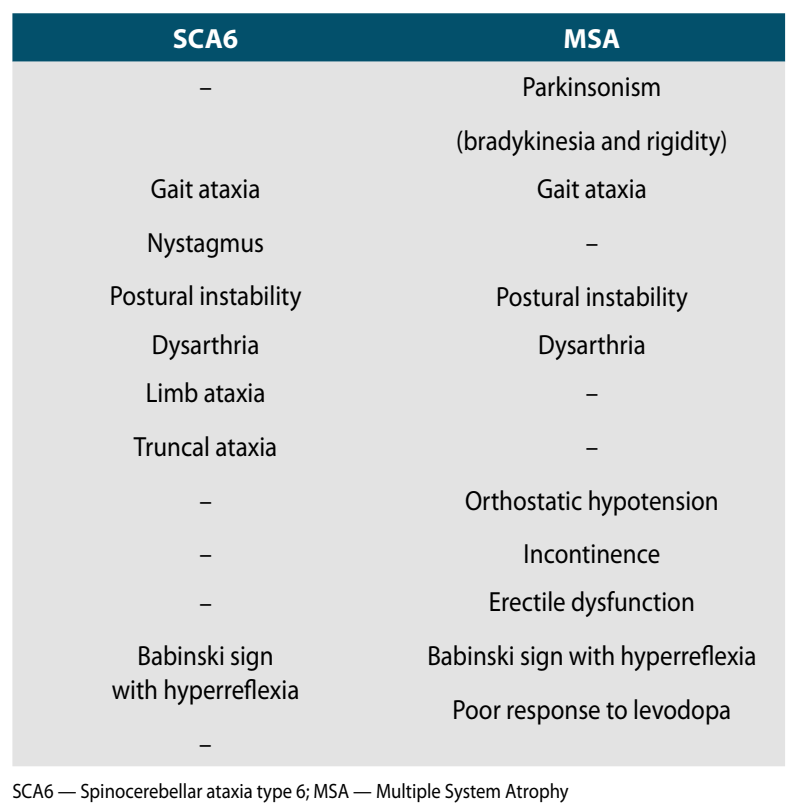

Head MRI revealed cerebellar atrophy, but no apparent pontine atrophy (Supplemental Fig. 1). The patient died 13 years later.

The autopsy report, performed at another institution, indicated atrophy of the dentate nucleus, substantia nigra, inferior olivary nucleus, and cerebellar cortex. Neuronal loss was evident in the subthalamic nucleus, globus pallidus, substantia nigra, and inferior olivary nucleus. Gliosis was present in the subthalamic nucleus, basal ganglia, globus pallidus, and putamen, and Purkinje cell loss was evident. It is unknown whether $a$-synuclein was present in brain pathology.

The patient's eldest son (subject IV-1, Fig. 1) developed loss of balance leading to falls at the age of 65 ; he was evaluated by a local neurologist, exhibited nystagmus and was then diagnosed with SCA6.

\section{Discussion}

Familial cases are more rarely encountered in MSA compared to SCA. Some familial forms of MSA have been reported previously in the literature. The first was reported in 1964, although the diagnosis of MSA is questionable considering the lack of pathology and the presence of atypical clinical features $[13,14]$. When 157 patients with possible and probable MSA were studied for the presence of family history of neurological conditions, only one was found to carry a family history of MSA [15]. Autosomal dominant inheritance of MSA was reported in a German family [16], and four families were reported to have autosomal recessive MSA [17]. This latter study discussed the presence of pontine atrophy and 'hot cross bun' sign on cranial MRI [17]; however, these findings can also be encountered, albeit infrequently, in patients with
SCA types 2 and 3 [17-19]. While the likelihood of a definitive diagnosis of MSA with pontine atrophy and 'hot cross bun' sign is decreased by these findings, it should remain a differential diagnosis given the reported cases of familial MSA.

Cerebellar ataxia, parkinsonism, pyramidal signs, and autonomic dysfunction are signs often shared between MSA and SCA patients [20]; while the first condition is sporadic and the second is a genetic autosomal dominant disease, both fall under spinocerebellar degeneration and exhibit olivopontocerebellar, striatal, and spinal cord involvement [12, 14]. Even with its autosomal dominant genetic disposition, there is a $15-20 \%$ chance that SCA may still occur sporadically, more commonly encountered among the trinucleotide forms of SCA [12]. Clinically differentiating between the two conditions can pose a challenge, especially during the early stages [21]. In a review of 203 patients with pathologically confirmed MSA, $110(54 \%)$ displayed cerebellar ataxia, $98(49 \%)$ pyramidal signs, $176(87 \%)$ parkinsonism, and 150 (74\%) presented with autonomic failure [22]. Parkinsonism was the most frequently reported form of motor impairment, followed by cerebellar ataxia [22]. Parkinsonism has been previously described in SCA6 patients [23], and one patient presented with parkinsonism and urinary incontinence and was initially diagnosed with MSA [24]. Our patient's initial symptoms included gait impairment, falls, and light-headedness; based on the clinical presentation, both SCA and MSA were considered.

In addition to the overlap in symptomatology (Tab. 1), disease onset tends to occur later in life with both MSA and SCA6 $[15,25]$. Mean age at onset for patients with SCA6 is 43 to 52 years (range 19-71) [5]; therefore, as expected, disease progression tends to be slower $[6,26]$. This was evident in our patient, whose onset of symptoms occurred at 68 years, with a disease duration of 15 years. Total lifespan remains unaffected in patients with SCA6 [5]. A retrospective analysis investigating disease progression and survival rates among different forms of cerebellar degenerative disorders revealed that the onset of MSA occurs between the ages of 50 and 60 , with a median of 56 years [27]. The study concluded that patients with MSA exhibited a faster rate of progression compared to SCA patients. Of note, the majority of the patients with SCA in this study had SCA type 1 , type 2 , or type 3; patients with SCA6 were not included as a group, given the low sample size $(\mathrm{n}=4)$ [27].

Distinguishing MSA from SCA is difficult in clinical practice due to the phenotypic overlap. Studies have investigated radiological features of MSA and SCA to create a standardised protocol in distinguishing the two conditions $[1,21]$. However, MRI findings commonly associated with MSA, such as the 'hot cross bun' sign, hyperintense putaminal rim, and olivopontocerebellar atrophy, can also be encountered with SCA $[12,28]$. 'Hot cross bun' sign and middle cerebellar peduncle hyperintensities were highly specific MRI findings in 12 pathologically confirmed MSA cases; they were identified in seven (58.3\%) and six (50\%) of the 12 respectively [29]. Our patient's MRI findings of cerebellar atrophy and lack of pontine involvement point 
toward a pure cerebellar condition. One study used magnetic resonance diffusion kurtosis imaging to monitor and identify pathological alterations in patients with cerebellar MSA or SCA, concluding that diffusion kurtosis imaging can be used when differentiating between the two conditions [1]. Another study measured peripheral neurofilament light levels in patients with MSA or SCA compared to controls, demonstrating a significant increase in neurofilament light levels in those with SCA [30].

Perhaps measuring neurofilament light levels could serve as a method to distinguish between the two conditions in future cases. Early and prompt diagnosis of MSA and SCA can improve prognosis, facilitate for better management and a more precise therapeutic plan $[12,28]$.

Genetic counselling is offered to all SCA patients and family members. Early detection allows patients planning for pregnancy to seek prenatal genetic testing as well as preimplantation genetic testing [5].

Our study is limited due to the lack of formal orthostatic and autonomic evaluations. However, other signs and symptoms present in our patient still point towards MSA as a possible diagnosis. MSA and SCA can have similar presentations during early stages, and the presence of both conditions on the list of differential diagnoses can be a common dilemma for clinicians. Further analysis will aid in developing a biomarker for longitudinal observations and follow up on potential therapies that undoubtedly will be developed. Genetic analysis is already widely utilised in SCA cases, and it can bring precision to the differential diagnosis.

\section{Clinical implications/future directions}

Despite the rare occurrence of MSA and SCA as differentials, reporting such cases is crucial. Their reporting in the literature will provide a stronger incentive to investigate and discover blood and cerebrospinal fluid biomarkers that will assist in future management.

Funding: This publication was prepared without any external source of funding. Mayo Clinic does not endorse specific products or services included in this article.

(C)2020 Mayo Foundation for Medical Education and Research Author contributions: Dr Hanna Al Shaikh: Organising, co-ordinating with family, study design, data acquisition, data analysis, drafting, and revising the manuscript. Ms. Wernick: Sample processing, laboratory analysis, and variant identification. Mrs. Strongosky: Participant identification and recruitment, co-ordinating family visits, and sample collection. Mrs. Soto-Beasley: Sample processing, laboratory analysis, and variant identification. Dr van Gerpen: Patient care and participant identification. Dr Cheshire: Patient care. Dr Uitti: Patient care. Dr Ross: Genetic testing, analysis and interpretation, study concept, study design, study supervision, and revising the manuscript. Dr Wszolek: Participant identification, study concept, study design, analysis and interpretation of data, study supervision, obtaining funding, and revising the manuscript.
Disclosures: Dr Hanna Al Shaikh: Receives funding from the Donald G. and Jodi P. Heeringa Family. Dr Ross: Is partially supported by R01-NS078086, P50-NS072187, U54 NS100693, U54 NS110435, Department of Defense, and the Michael J. Fox Foundation. He is an editorial board member for the American Journal of Neurodegenerative Disease, Frontiers in Neurology: Neurogenetics, and Molecular Neurodegeneration. Dr Wszolek: Is partially supported by the Mayo Clinic Centre for Regenerative Medicine, gifts from The Sol Goldman Charitable Trust, and the Donald G. and Jodi P. Heeringa Family, the Haworth Family Professorship in Neurodegenerative Diseases Fund, and the Albertson Parkinson's Research Foundation. He serves as PI or Co-PI on grants from Abbvie, Inc (M15-562 and M15-563), Biogen, Inc (228PD201), and Biohaven Pharmaceuticals, Inc (BHV4157-206 and BHV3241-301). He serves as PI of the Mayo Clinic American Parkinson's Disease Association Information and Referral Centre. Dr Cheshire, Ms. Wernick, Mrs. Strongosky, Mrs. Soto-Beasley, Drvan Gerpen and Dr Uitti report no disclosures. Acknowledgments: We would like to thank our patient and her family for their co-operation and support for this research study. We would also like to thank our collaborators and partners in the lab for taking the time to verify all blood samples and their continuous efforts in this research endeavour. Mayo Clinic is an American Parkinson's Disease Association (APDA) Information and Referral Centre, an APDA Centre for Advanced Research, and a Lewy Body Dementia Association Research Centre of Excellence. Dr. Wszolek serves as Co-Editor-in-Chief of Neurologia $i$ Neurochirurgia Polska. This manuscript processing was performed by Dr. Sławek, the second Co-Editor-in-Chief of journal.

\section{References}

1. Ito, K. Differentiation Between Multiple System Atrophy and Other Spinocerebellar Degenerations Using Diffusion Kurtosis Imaging. Acad Radiol, 2019. 26(11): p. : e333-e339.

2. Hanna AI Shaikh R, Caulfield T, Strongosky AJ, et al. TRIO gene segregation in a family with cerebellar ataxia. Neurol Neurochir Pol. 2018; 52(6): 743749, doi: 10.1016/j.pjnns.2018.09.006, indexed in Pubmed: 30279051.

3. Fujioka S, Sundal C, Wszolek ZK. Autosomal dominant cerebellar ataxia type III: a review of the phenotypic and genotypic characteristics. Orphanet J Rare Dis. 2013; 8: 14, doi: 10.1186/1750-1172-8-14, indexed in Pubmed: 23331413.

4. Whaley NR, Fujioka S, Wszolek ZK. Autosomal dominant cerebellar ataxia type I: a review of the phenotypic and genotypic characteristics. Orphanet J Rare Dis. 2011; 6: 33, doi: 10.1186/1750-1172-6-33, indexed in Pubmed: 21619691.

5. Casey, H.L. and C.M. Gomez, Spinocerebellar Ataxia Type 6, in GeneReviews((R)), M.P. Adam, , Editors. 1993: Seattle. (WA).

6. Buijsen RAM, Toonen $\sqcup A$, Gardiner SL, et al. Genetics, Mechanisms, and Therapeutic Progress in Polyglutamine Spinocerebellar Ataxias. Neurotherapeutics. 2019; 16(2): 263-286, doi: 10.1007/s13311018-00696-y, indexed in Pubmed: 30607747.

7. Ishikawa K, Tanaka H, Saito M, et al. Japanese families with autosomal dominant pure cerebellar ataxia map to chromosome 19p13.1-p13.2 and are strongly associated with mild CAG expansions in the spinocerebellar ataxia type 6 gene in chromosome 19p13.1. Am 
J Hum Genet. 1997; 61(2): 336-346, doi: 10.1086/514867, indexed in Pubmed: 9311738.

8. Shizuka M, Watanabe M, lkeda Y, et al. Molecular analysis of a de novo mutation for spinocerebellar ataxia type 6 and (CAG)n repeat units in normal elder controls. Journal of the Neurological Sciences. 1998; 161(1): 85-87, doi: 10.1016/s0022-510x(98)00270-6.

9. Mariotti C, Gellera C, Grisoli M, et al. Pathogenic effect of an intermediate-size SCA-6 allele (CAG)(19) in a homozygous patient. Neurology. 2001; 57(8): 1502-1504, doi: 10.1212/wnl.57.8.1502, indexed in Pubmed: 11673601.

10. Yabe I, Sasaki H, Matsuura T, et al. SCA6 mutation analysis in a large cohort of the Japanese patients with late-onset pure cerebellar ataxia. Journal of the Neurological Sciences. 1998; 156(1): 89-95, doi: 10.1016/s0022-510x(98)00009-4.

11. Gilman S, Low PA, Quinn N, et al. Consensus statement on the diagnosis of multiple system atrophy. Journal of the Neurological Sciences. 1999; 163(1): 94-98, doi: 10.1016/s0022-510x(98)00304-9.

12. Gilman, S., , Second consensus statement on the diagnosis of multiple system atrophy. Neurology, 2008. 71(9): p. : 670-6.

13. Lewis, P., Familial Orthostatic Hypotension. Brain, 1964. 87: p. : 719-28.

14. Quinn N. Multiple system atrophy-the nature of the beast. J Neurol Neurosurg Psychiatry. 1989; Suppl: 78-89, doi: 10.1136/jnnp.52. suppl.78, indexed in Pubmed: 2666581.

15. Soma, H., , Heredity in multiple system atrophy. J Neurol Sci, 2006. 240(1-2): p. : 107-10.

16. Wüllner U, Abele M, Schmitz-Huebsch T, et al. Probable multiple system atrophy in a German family. J Neurol Neurosurg Psychiatry. 2004; 75(6): 924-925, doi: 10.1136/jnnp.2003.025155, indexed in Pubmed: 15146018.

17. Hara $\mathrm{K}$, Momose $\mathrm{Y}$, Tokiguchi $\mathrm{S}$, et al. Multiplex Families With Multiple System Atrophy. Archives of Neurology. 2007; 64(4): 545, doi: 10.1001/archneur.64.4.545.

18. Bürk K, Skalej M, Dichgans J. Pontine MRI hyperintensities ("the cross sign") are not pathognomonic for multiple system atrophy (MSA). Mov Disord. 2001; 16(3): 535, doi: 10.1002/mds.1107, indexed in Pubmed: 11391754.

19. Murata $\mathrm{Y}$, Yamaguchi S, Kawakami H, et al. Characteristic magnetic resonance imaging findings in Machado-Joseph disease. Arch Neu- rol. 1998; 55(1): 33-37, doi: 10.1001/archneur.55.1.33, indexed in Pubmed: 9443709.

20. Yeh TH, Lu CS, Chou YH, et al. Autonomic Dysfunction in Machado-Joseph Disease. Archives of Neurology. 2005; 62(4): 630, doi: 10.1001/ archneur.62.4.630.

21. Kim, M., , Differential value of brain magnetic resonance imaging in multiple system atrophy cerebellar phenotype and spinocerebellar ataxias. Sci Rep, 2019. 9(1): p. ; 17329.

22. Wenning GK, Tison F, Shlomo Yb, et al. Multiple system atrophy: A review of 203 pathologically proven cases. Movement Disorders. 1997; 12(2): 133-147, doi: 10.1002/mds.870120203.

23. Park H, Kim HJ, Jeon BS. Parkinsonism in spinocerebellar ataxia. Biomed Res Int. 2015; 2015: 125273, doi: 10.1155/2015/125273, indexed in Pubmed: 25866756.

24. Frequency Analysis and Clinical Characterization of Spinocerebellar Ataxia Types 1, 2, 3, 6, and 7 in Korean Patients-Correction. Archives of Neurology. 2003; 60(9): 1256, doi: 10.1001/archneur.60.9.1256.

25. Murata Y, Kawakami H, Yamaguchi S, et al. Characteristic magnetic resonance imaging findings in spinocerebellar ataxia 6. Arch Neurol. 1998; 55(10): 1348-1352, doi: 10.1001/archneur.55.10.1348, indexed in Pubmed: 9779664.

26. Ashizawa T, Öz G, Paulson HL. Spinocerebellar ataxias: prospects and challenges for therapy development. Nat Rev Neurol. 2018; 14(10): 590605, doi: 10.1038/s41582-018-0051-6, indexed in Pubmed: 30131520.

27. Klockgether T, Lüdtke R, Kramer B, et al. The natural history of degenerative ataxia: a retrospective study in 466 patients. Brain. 1998; 121 ( Pt 4): 589-600, doi: 10.1093/brain/121.4.589, indexed in Pubmed: 9577387.

28. Li M, Ma Q, Zhao X, et al. Dilemma of multiple system atrophy and spinocerebellar ataxias. J Neurol. 2018; 265(12): 2764-2772, doi: 10.1007/s00415-018-8876-x, indexed in Pubmed: 29700645.

29. Massey LA, Micallef C, Paviour DC, et al. Conventional magnetic resonance imaging in confirmed progressive supranuclear palsy and multiple system atrophy. Mov Disord. 2012; 27(14): 1754-1762, doi: 10.1002/mds.24968, indexed in Pubmed: 22488922.

30. Wilke C, Bender F, Hayer SN, et al. Serum neurofilament light is increased in multiple system atrophy of cerebellar type and in repeat-expansion spinocerebellar ataxias: a pilot study. J Neurol. 2018; 265(7): 1618-1624, doi: 10.1007/s00415-018-8893-9, indexed in Pubmed: 29737427. 